\title{
Research on the Application of TRIMP Method in the Training of Martial arts Athletes
}

\author{
Yong Ding, Shuai Liu \\ Pingxiang University, Pingxiang, Jiangxi, 337055, China
}

\begin{abstract}
Keywords: TRIMP; Martial arts; Training monitoring
\end{abstract}
\begin{abstract}
Based on impulse training heart rate, TRIMP has a range of individual standard, through the strength of sports and the movement time to represent the different range of training load. The testing method is simple, and noninvasive, the results of evaluation are scientific, intuitive. TRIMP also helps to solve the shortcomings of conventional biochemical indicators. This paper firstly discusses the influence of different methods on the martial arts teaching of male athletes. In this paper, the Anhui martial arts team is used as the research object, to explore the application of TRIMP in martial arts training monitoring, to find a simple and effective means of training and monitoring of martial arts.
\end{abstract}

\section{Introduction}

Chinese martial arts have a long history, and are rich in content and form. Routine sports includes boxing and instruments, Fighting activities such as Kung Fu have free combat and grappling, Chinese martial arts has its unique performance and fitness function, which is becoming the majority of the people love the project. Although the training athletes have strong interest in learning, but because of the differences in sports coupled with martial arts, training results are often unsatisfactory. How to mobilize the enthusiasm of the athletes in the training time, training the ability of autonomous learning, improve teaching efficiency and quality, is a common topic of martial arts training in Colleges and universities [1-3].

Martial arts fight belongs to non confrontational projects, the volume and intensity loading of Wushu is influenced by competition of physical endurance, the level of competition, competition environment. Various factors influence the subjective effort of how to make athletes, coaches convenient and true understanding of the athletes' training load, to provide a scientific basis to develop training programs for coaches, which is an important aspect of sports scientific research [4]. In the long-term research of competitive sports, we often find that biochemical monitoring methods, such as hemoglobin (HB), blood urea (BUN), Creatine kinase (CK), serum testosterone (T), compound $\mathrm{F}(\mathrm{C})$, although it can reflect the training and match the load to a certain extent, but because the test itself is a trauma, which cannot limit the sampling, mostly only as a means of monitoring the stage, the training content for a short period of time such as individual teaching. Monitoring the single game is difficult to achieve accurate monitoring. In addition, the biochemical index is a comprehensive reflection of the athletes' function, test results is not only related to exercise training, but also is influenced by many factors, such as the HB and the amount of water on the athletes, and athletes in the diet intake of protein BUN, CK and so on [5]. In practice, the test results are often inconsistent with the coaches and athletes' subjective feeling, so it is necessary to do a lot of screening work if we want to use the above indicators to reflect the training load. Heart rate changes with the psychological stress exercise time, exercise intensity. Heart rate also changes the energy in the training to match the load intensity and loads well reflect the athletes training, which is based on the impulse of heart rate (TRIMP).

\section{Relevant method}

\subsection{Training monitoring content}

The athletic training state and nutrition regulation are always inseparable, in order to cooperate with the training of athletes, athletes adjust the function state, which is consistent with nutrition 
athletes, including blood source, power source, powerful polypeptide, glutamine, fructose, sports drinks. Test training monitoring indexes related to the blood and urine routine test, such as BU, CK, T, C, LA, electrocardiogram, dynamic force, anaerobic power. Martial arts team has the training venue and different competition characteristics, the amount of training lasts 8-10 months, and we arrange the training of the teams every night, our training for athletes after training monitoring are collected on the same day or the second day morning while the results feedback timely, in order to grasp the coach athlete status at any time, we can timely adjust the training plan $[6,7]$.

\subsection{TRIMP computing method}

In order to simplify the method of quantifying training load, Foster and their colleagues have proposed a method to evaluate training load using the Borg RPE scale (the Borg of Perceived Exertion scale). This RPE is based on TRIMP computing model, the training period of time (minutes) multiplied by $R P E$ scores. For example, during 60 minutes of weight training, grade is very $\operatorname{difficult}(R P E=7)$,

$$
\text { TRIMP }=60 * 7=420
$$

Table 1 . The degree of perceived exertion rating scale

\begin{tabular}{|c|c|c|c|}
\hline E & Descriptor & Rating & Descriptor \\
\hline 0 & Rest & 6 & - \\
\hline 1 & Very, very easy & 7 & Very hard \\
\hline 2 & Easy & 8 & - \\
\hline 3 & Moderate & 9 & - \\
\hline 4 & Somewhat hard & 10 & Maximal \\
\hline E & Hard & & \\
\hline
\end{tabular}

In this paper, the reliability of TRIMP method was studied, and the subjects were given different types of aerobic training on the training ground, and then after 30 minutes of training, the intensity of training activity was evaluated. Although the $R P E$ scale used in the experiment was not a traditional scale, they did not affect the intensity of the exercise [8]. The Foster's study showed that $R P E$ was a more reliable indicator of heart rate during the evaluation of endurance training.

\section{Research objects and methods}

\subsection{Research objects}

Twenty athletes of Anhui province martial arts team are used as the research object, which is separated into two classes by the national level of athletes, every one of who has an average training period of more than 10 years. 
Table 2. Basic motion information

\begin{tabular}{|c|c|c|c|c|}
\hline Sex & NO. & Age & height & weight \\
\hline male & 10 & $22.48 \pm 1.56$ & $187.37 \pm 3.23$ & $79.39 \pm 4.47$ \\
\hline female & 10 & $20.33 \pm 1.97$ & $172.48 \pm 2.8$ & $52.48 \pm 1.72$ \\
\hline
\end{tabular}

\subsection{Research methodology}

\section{Experimental method}

A total of twenty male athletes are randomly selected as subjects, and the results are compared with those of before and after the training by using the special strength training program designed in this study. At the same time, the relevant experts shall be invited to conduct a comprehensive audit and evaluation of the test index. The experimental scheme helps to ensure the effective selection of the experimental index and the experimental scheme. The continuous heart rate record of athletes in training and national competition produced in Finland, and its sampling frequency is set as 1 second.

2. TRIMP method

We adopt the training area stimulation method, according to the individual maximum heart rate, the players can be divided into five levels.

3. Research contents

In order to obtain the ideal special quality training effect, the training object should be considered in combination with the technical action and the different training methods [1]. To achieve the effect of strength training, it must be combined with action different choice of training methods. First of all, TRIMP can not only make the inverse dynamic role in the muscles get exercise, but also can be helpful for the improvement of the relationship between the central coordination. Due to the different action in muscles are not the same, the key link in the training must be good at analysis of technical movements, so as to correctly determine the major muscles in sports, and carry out targeted training to develop appropriate training methods. For example, a variety of movements in martial arts is accomplished through the waist force, thus to make the training methods on the development of waist muscle strength, increased waist strength and flexibility through the abdominal and leg kick, such as exercise, the muscles are all involved in the action of coordinated development.

1. Experimental group of athletes to carry out specific strength training methods

Long term training practice has proved that, through the accurate understanding of the various forces related to technical movements, the use of effective strength training methods, the training effect will often be greatly improved. The following is the specific training methods of Wushu routine athletes.

(1) The explosive upper shoulder and elbow muscles mainly triceps commonly used flat bench press, dumbbell push, stopped with a long pole training method.

(2) The core strength includes the main spine muscles of rectus abdominis muscle, internal oblique, external oblique, which generally use the sit ups swivel and hold weights swivel. The core strength also pulls on the side of the body by lifting bell and throwing medicine ball, and also flexes training collection method body upside down on.

(3) General power. The main role of shoulder, hip and knee joint muscles of major muscle often used to do all kinds of martial arts, which need the player fast, clean and the snatch hold jump, through a continuous complete martial arts action training method.

(4) Boxing Routine Power. Single or combined action strength training needs to complete the action repeatedly. In order to increase the intensity of boxing, foot and flight height, a full set of actions by weight method and repetition alternate training, improve the training motion force and special endurance. 
(5) Power equipment routine complete single or combined action by overload training method, and increase the equipment length, the practice is mainly the use of equipment speed and strength. Completing the action is the intensity of the whole set of apparatus movement training overload condition and resistance.

2. Wushu special strength training of the amount of exercise arrangements

Martial arts need that the athlete have special strength training time of about 2 hours each time, the training objectives of the arrangement of the 3- exercises can be 10. In the strength training, we must focus on speed and strength training. When the strength training is carryied on, the speed of the middle and small weight is high. According to the need, sometimes we can also carry on the strength training of large weight is the weight of the maximum weight of $70 \%-90 \%$, each group to $3-5$ times is appropriate, focusing on the development of absolute power. In endurance training, routine endurance training with body weight helps to complete the bare handed routines, or to increase the weight of the equipment to complete the training of equipment routines. In order to improve the training effect, the 2- 3 sets of movements can be completed continuously, so that the endurance level of athletes can be improved under the stimulation of overload.

Table 3. Comparison of the whole technology and the level of training before and after the experiment

\begin{tabular}{|c|c|c|c|c|}
\hline Time & Level & Movement Quality & $\begin{array}{c}\text { Action } \\
\text { Difficulty }\end{array}$ & $\begin{array}{l}\text { Full set of } \\
\text { Technology }\end{array}$ \\
\hline & Mean \pm SD & Mean \pm SD & Mean \pm SD & Mean \pm SD \\
\hline $\begin{array}{c}\text { Before } \\
\text { exp }\end{array}$ & $2.64 \pm 0.09$ & $4.68 \pm 0.12$ & $1.36 \pm 0.11$ & $8.59 \pm 0.29$ \\
\hline $\begin{array}{l}\text { After } \\
\exp \end{array}$ & $2.81 \pm 0.08$ & $4.89 \pm 0.07$ & $1.62 \pm 0.13$ & $9.28 \pm 0.24$ \\
\hline $\mathrm{T}$ & 4.12 & 3.10 & 4.06 & 3.98 \\
\hline $\mathrm{P}$ & $<0.01 * *$ & $<0.05^{*}$ & $<0.05^{*}$ & $<0.01 * *$ \\
\hline
\end{tabular}

4. Research results

1. training class TRIMP

Record the continuous heart rate of each of the 10 players in the men's Wushu group, According to the above method to calculate the TRIMP of each lesson, athletes fasting venous blood test BU results is shown in Fig. 1.

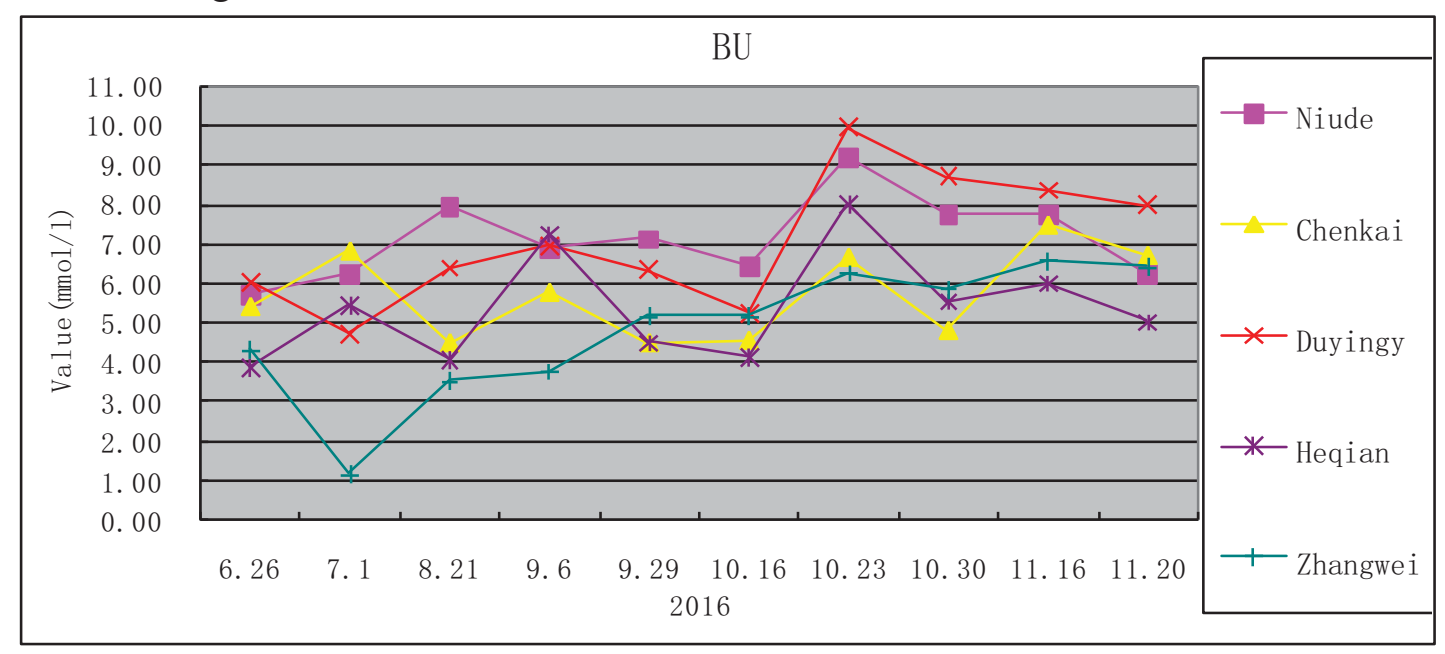

Fig. 1. Athletes fasting venous blood test BU results 
We can get the following conclusions through figure 1 . The training plan is almost identical, 10 athletes in the same class by TRIMP are not the same, such as the TRIMP Monday morning for a minimum of 118.9, up to 465.5. Different athletes in the training performance are different, although some athletes participate in training, high intensity exercise in the same time. Some athletes for various reasons do not go into training, then the same time athletes low strength, two athletes coaches evaluation of the training effect are obviously different. The load of athletes in different training courses in the same change trend everyday. The load of each lesson is regular wave shape, which is helpful to promote the recovery and improve the training effect.

\section{Summary}

After 20 weeks experimental research we can get that there were significant differences before and after the experiment of 20 male physical arts athletes who have special strength quality level, especially in basic skills, martial arts movement strength and movement technology. Overall performance level and the difficulty of movement have different degree to improve, which compared with he training before training monitoring.

1. TRIMP value can reflect the training content, $\mathrm{BU}$ and $\mathrm{CK}$ are used to reflect the athlete's response to training.

2. Compared with the commonly used biochemical indicators, TRIMP could more external load and internal load of athletes to fully reflect the bout.

3 Through the comparative study of the TRIMP value of the teaching competition and the national competition, it is found that the load in the teaching competition is far less than the load in the national competition, and the training can not meet the actual requirements.

\section{References}

[1] WANG, Cong, and Xuelin QIN. Monitoring Training and Competition Load based on TRIMP in Badminton [J]. Sports \& Science 6 (2013): 015.

[2] Gubbels J, van der Stouwe T, Spruit A, et al. Martial arts participation and externalizing behavior in juveniles: A meta-analytic review[J]. Aggression and violent behavior, 2016, 28: 73-81.

[3] Miracle J. Imposing the terms of the battle: Donn F. Draeger, Count Dante, and the struggle for American martial arts identity[J]. Martial Arts Studies, 2015 (1): 46-59.

[4] De Guzman J. Aspire: a creative exploration of the short, lyrical documentary[D]. Auckland University of Technology, 2013.

[5] Marchetti, Gina. Does the Karate Kid Have a Kung Fu Dream? Hong Kong Martial Arts; between Hollywood and Beijing [J]. JOMEC Journal 5 (2016).

[6] Wong, Wayne KT. "Synthesizing Zhenshi (authenticity) and Shizhan (combativity): reinventing Chinese kung fu in Donnie Yen's Ip Man series (2008-2015) [C]." Martial Arts Studies 3 (2017): 71-88.

[7] Miracle, J. (2015). Imposing the terms of the battle: Donn F. Draeger, Count Dante, and the struggle for American martial arts identity [M]. Martial Arts Studies, (1), 46-59.

[8] Wile, D. (2016). Asian Martial Arts in the Asian Studies Curriculum [M]. JOMEC Journal, (5).

[9] Shuman, Amanda. The Politics of Socialist Athletics in the People's Republic of China, 1949-1966 [J]. (2014). 